CAPÍTULO 2

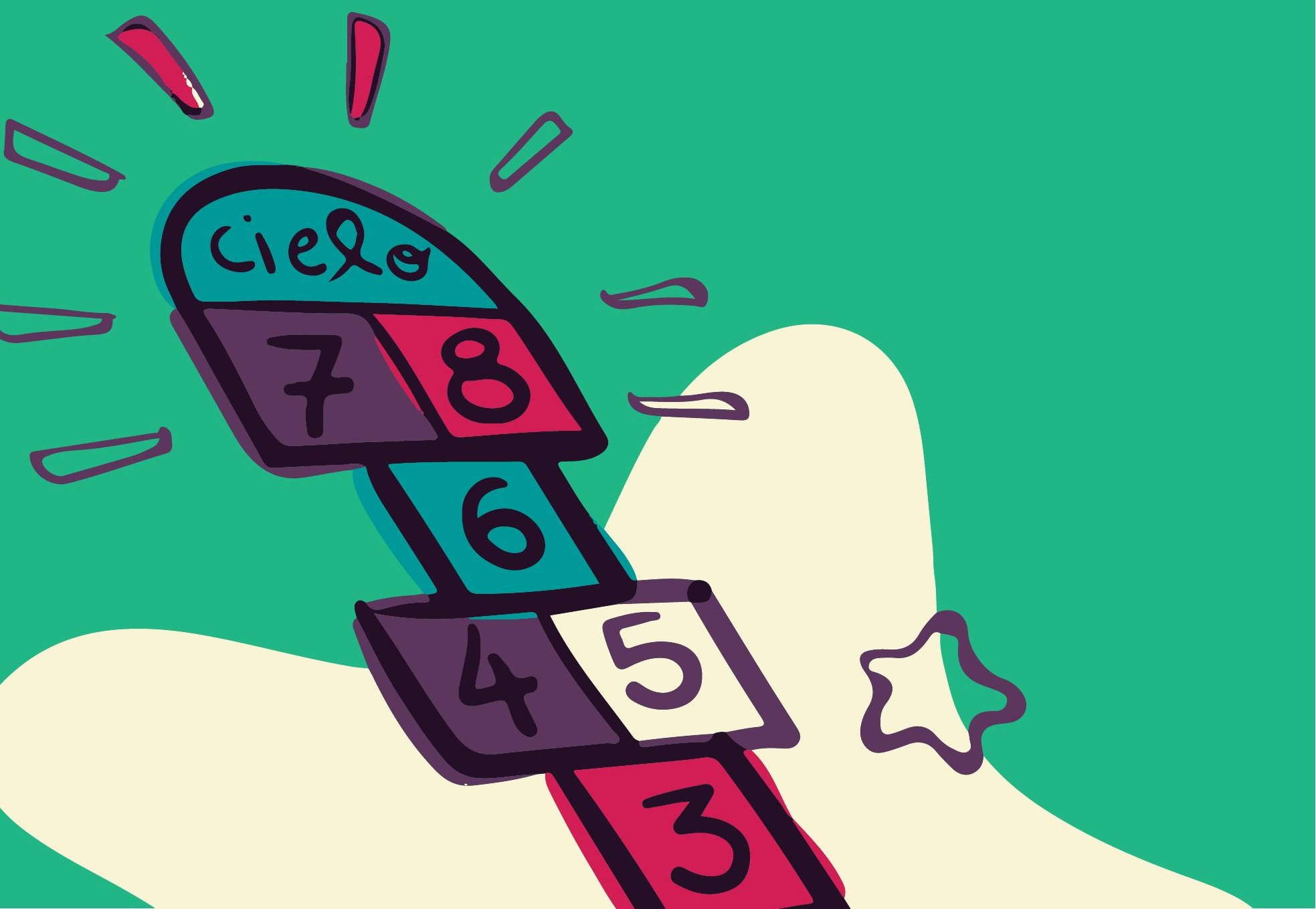




\section{La importancia de evaluar el impacto de las ludotecas en Colombia}

En este capítulo, el lector encontrará el desarrollo del proceso que ha surtido la Corporación Juego y Niñez en la línea de investigación, sistematización y gestión del conocimiento para la mejora continua de sus proyectos $y$, en especial, en torno al modelo

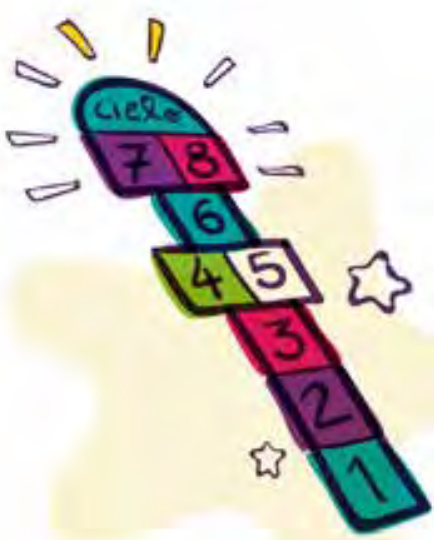
de Ludotecas Naves (Niños y niñas, Aprendiendo, Vivenciando, Experimentando y Socializando), proceso que será la principal razón por la cual la Corporación Juego y Niñez decide, entre los años 2014-2018, contratar con la Universidad Nacional de Colombia la primera investigación longitudinal en Colombia sobre el impacto de las ludotecas Naves en el desarrollo de competencias ciudadanas, emocionales y de creatividad. 
Partiremos de narrar, de manera simultánea, cómo evoluciona el programa de ludotecas Naves en Colombia y cómo, en la Corporación, investigar, sistematizar y transferir conocimiento centrando sus intereses en la reflexión y análisis ha sido fundamental para los impactos que hemos alcanzado 20 años después de su inicio en 1999 en Colombia. Este modelo de implementar ludotecas Naves en más de 100 municipios del país y abrir el camino para que más de 400 ludotecas en Colombia- a veces llamadas de otra manera- se hayan instalado en parques, plazas, polideportivos, casas, recreos, hospitales, cárceles y calles de todo el país, ha sido un reto que muestra resultados, porque se logró que, desde posturas políticas, poniendo la niñez en el centro, dándole un lugar al juego con sentido como fin, los recursos públicos y privados se hayan mantenido como política local en favor de la infancia y adolescencia, asegurando su calidad, por supuesto, con muchas tensiones por su sostenibilidad.

\section{Sistematizar para definir un modelo}

Al poco tiempo de iniciado el desarrollo de las ludotecas Naves por la Corporación Juego y Niñez, resultado de la observación, evaluación y operación, para su estructura administrativa y técnica se definen roles y responsabilidades de los diversos actores interesados en el programa y es así como se propone un modelo desde corresponsabilidades y responsabilidades del desarrollo de la infancia y adolescencia en cada territorio (gráfico 1). Sustenta cómo los recursos privados, desde la experticia de la Corporación, se convierten en el motor inicial para que una ludoteca sea viable, se ponga en marcha y se acompañe técnicamente, por un tiempo, para así dar lugar a que actores locales estatales definan la ludoteca desde una de sus secretarías, para ponerla al servicio del mayor número de participantes, tanto niños como adultos.

Un programa con la niñez en el centro- como ambiente protector-y en donde los gobiernos locales contratan profesionales, que forman y acompañan 


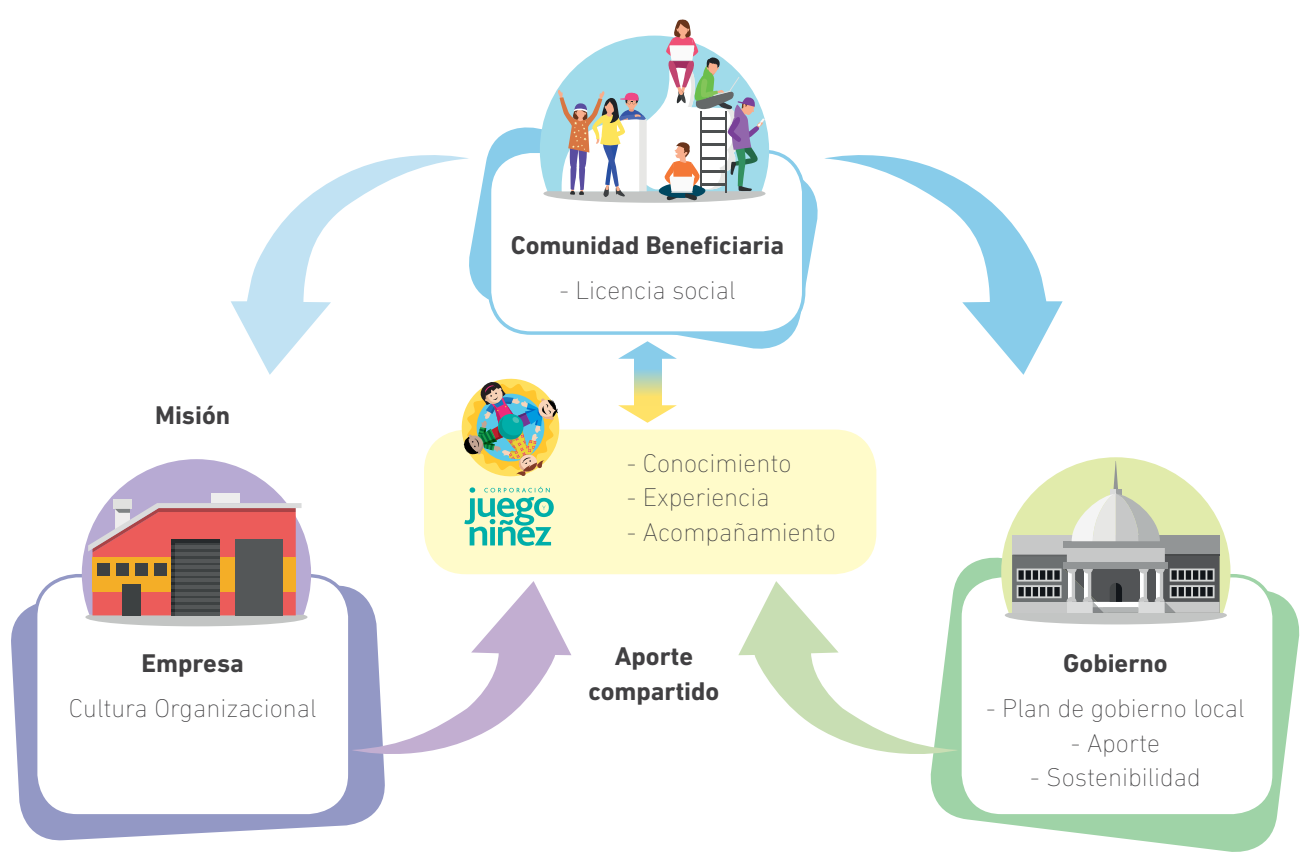

en la Corporación, para que desde el punto de vista metodológico, faciliten, animen, provoquen, propongan, generen reflexión, activen la imaginación y potencien la creatividad, logrando ambientes sociales en donde las expresiones como el juego, es una gran apuesta porque enriquecen la capacidad para potenciar, reconocer y comprender las distintas expresiones de niños, niñas y sus familias. Al llegar a los 20 años de implementar el modelo podemos afirmar que cuando cada actor público o privado asume su rol y responsabilidad, y la sostiene, se 
logra el disfrute pleno de la niñez en ambientes -con perspectiva de derechospara leer, pintar, crear, desarmar, saltar, cantar, conversar, reír y hasta llorarcon un lenguaje cotidiano de respeto, acuerdos, observación, mediación, “subiendo el adulto" al nivel de la mirada de la niñez.

Se han presentado diversas tensiones en su implementación, porque la imposibilidad de sostener recursos tanto de algunos aportantes de empresas privadas como de algunas administraciones municipales, ponen en riesgo asuntos vitales en el cumplimiento de estándares de calidad, tales como la remuneración necesaria del perfil del profesional ludotecario, mantenimiento de la infraestructura, transporte permanente para ludotecas viajeras a periferia y ruralidad, y la continua actualización del personal que los motive a innovar, aumentar cobertura, trascender grupos etarios, entre otros. Tensiones superadas la mayoría de las veces por los ludotecarios, quienes juegan un papel fundamental en la sostenibilidad del programa porque comprenden que su postura política debe ser desde la niñez como sujeto de derechos y sus aprendizajes se internalizan desde la gestión del conocimiento que fortalecen por su experiencia cotidiana en cada entorno diferente y único. Son muchos actores públicos y privados los que están sustentando la calidad de Las Ludotecas Naves como un modelo público- privado- después de 20 años de inicio en diversos departamentos de Colombia. Modelos que se han alimentado por procesos investigativos, de sistematización y de gestión de conocimiento, de los cuales la Corporación Juego y Niñez aprende de manera continua y reconoce que el modelo es posible porque ha contado con la sostenibilidad de recursos provenientes de empresas privadas, convocadas por los despachos de las esposas de los presidentes del país. 


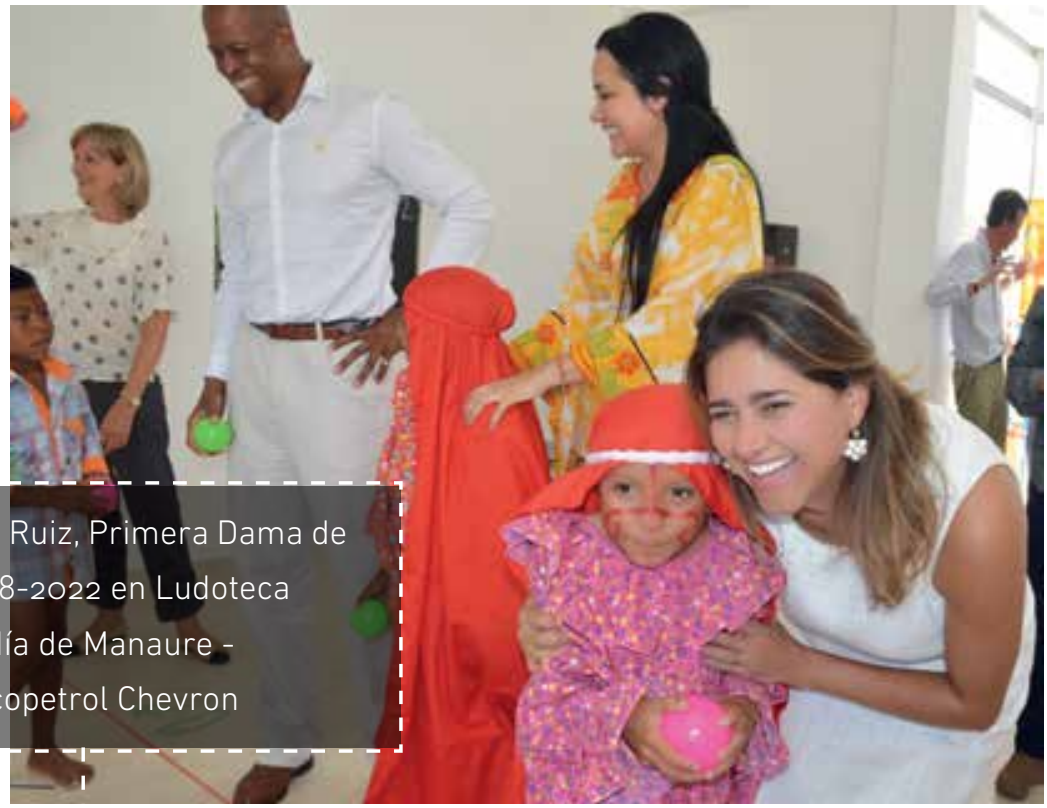

María Juliana Ruiz, Primera Dama de

Colombia 2018-2022 en Ludoteca

NAVES Alcaldía de Manaure -

Asociación Ecopetrol Chevron

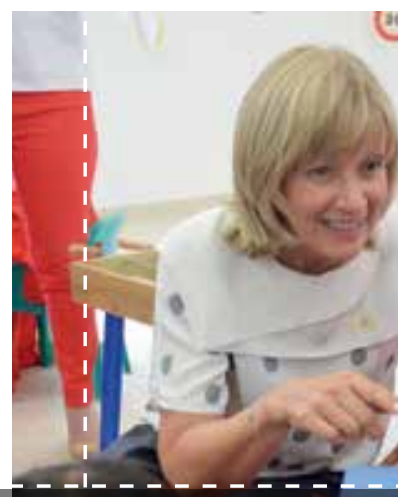

(ن)
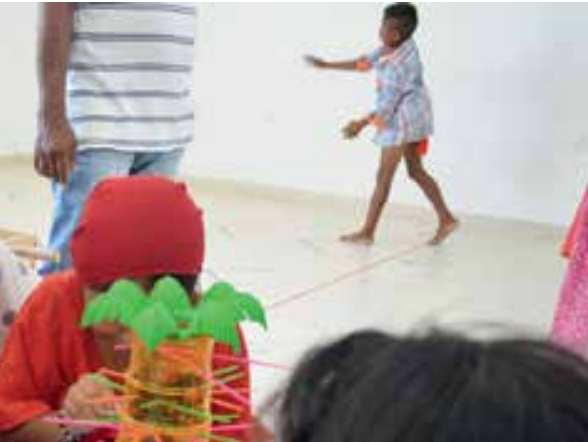

Nohora Puyana de Pastrana, Primera

Dama de Colombia 1998-2002 en

Ludoteca NAVES Alcaldía de Manaure

- Asociación Ecopetrol Chevron

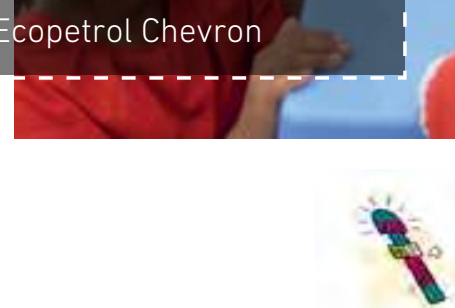

51 


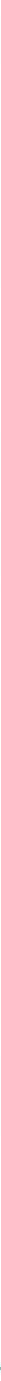


Es así como recursos de organizaciones privadas como Cerrejón, Microsoft, Petrobras, Chevron-Asociación Ecopetrol y Chevron, Colsubsidio, Cafam, Bayer, Pepe Ganga, Politécnico Grancolombiano, Nutresa, Nestlé, Coca Cola, Femsa-Coca Cola, Argos, Procter\&Gamble, Mattel, Arroz Casanare, McDonalds y Fundación Tras La Perla, entre otras, y compromisos y políticas públicas con prioridad en la infancia y adolescencia, han logrado que profesionales ludotecarios y actores locales, como alcaldes, secretarios, gobernadores, y concejales, estén acompañados por la Corporación Juego y Niñez para que, desde su proyecto de vida, porten con gran fuerza y color la camiseta de Ludotecas Naves y, siendo funcionarios públicos, hagan primar el rol de su profesión por encima de una postura partidista, demostrando que cuando la niñez es reconocida como sujeto de derechos, los avances son inmensos, como lo mostrará este libro en los resultados de la investigación longitudinal, y que se desarrollará en los capítulos siguientes.

\section{Investigaciones para la Metodología Naves}

Continuando con la línea de investigación, como constante en la Corporación Juego y Niñez, entre los años 2006 y 2008 se realizan dos procesos seguidos de investigación; el primero de tipo dramático con la Universidad Santo Tomás, y otro con un grupo de investigadores externos basados en la investigación de acción participativa. Desde ambos procesos, se recorrieron más de 20 ludotecas Naves que, para ese momento, se habían puesto en marcha y se logró darle volumen a las voces y sentires de quienes cada mañana llegaban a las ludotecas a generar lazos y huellas imborrables y vitales para el desarrollo de niños y niñas. Estas sistematizaciones desarrollaron una propuesta de metodología propia para que la Corporación Juego y Niñez, desde la experiencia de la cotidianidad de los territorios, contara con un norte para abordar la atención y mantener la calidad en una ludoteca Naves acompañada técnicamente u operada directamente por la Corporación. 
Como se explica en el gráfico 2, la Metodología Naves se convierte en ese derrotero cotidiano para que cada programa, proyecto, acción o intervención que desarrolla en el país la Corporación, cuente con ese sello de calidad al lograr realizarlo aplicando sus posturas, componentes y acciones. Desde ludotecas Naves, Celebración Día de la Niñez o cada nuevo programa que propone con otros sectores -salud, medio ambiente, educación y vivienda, entre otros-, aporta a cada colaborador o actor participante, como modificar las formas de ser y estar de los participantes en la relación consigo mismo, con el otro y con el entorno.

Esta Metodología propia, que cuenta con su debido registro de derechos de autor de la Corporación Juego y Niñez, explica a quienes quieren asumirla que es necesario adoptar al menos tres posturas, movilizar componentes estratégicos y propiciar su operación con buenas prácticas, de manera que se logre comprender el rol y calidad de quien se debe poner al frente del juego con la infancia y la adolescencia: a) Postura Política: respecto a reconocer el niño y la niña como sujetos de derechos para ejercer su participación, autonomía y ciudadanía; b) Postura Teórica: que aporta todos los argumentos sobre porqué el juego es una experiencia creativa, una necesidad vital, una acción libre particular y colectiva, y el desarrollo infantil, un proceso no lineal; c) Postura Estratégica: prueba que la articulación de actores es esa constante para activar y movilizar a las comunidades desde sus necesidades, así como incidir en los espacios políticos sobre la garantía de los derechos de la infancia.

\section{Sistematizar, investigar, gestión de conocimiento: una constante}

Cada programa y proyecto que la organización desarrolla en el país -ya sea de corto aliento o de más de tres años- mantiene la constante de revisarse permanentemente desde la sistematización e investigación. Mantener alianzas con la academia es un indicador que da cuenta del mejoramiento constante de sus inter- 


\section{GRÁFICO 2. METODOLOGÍA NAVES}
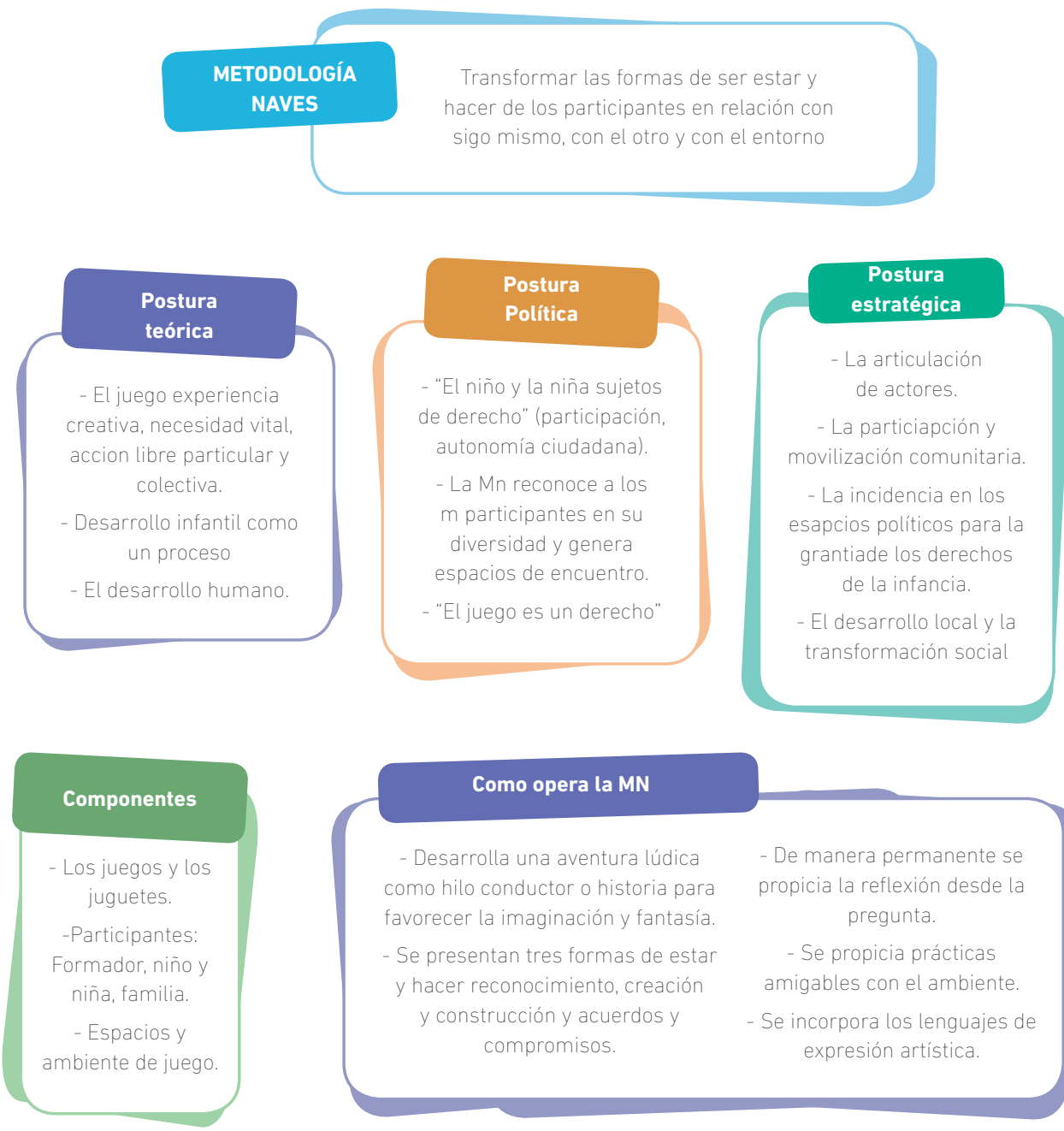

\section{Como opera la MN}

- Desarrolla una aventura lúdica como hilo conductor o historia para favorecer la imaginación y fantasía.

- Se presentan tres formas de estar y hacer reconocimiento, creación

y construcción y acuerdos y compromisos.
- De manera permanente se propicia la reflexión desde la

$$
\text { pregunta. }
$$

- Se propicia prácticas amigables con el ambiente.

- Se incorpora los lenguajes de expresión artística. 
venciones en los territorios. Cada sistematización y evaluación que la Corporación ha realizado le ha permitido aprender y trascender, por cuanto cada experiencia implementada se ha convertido, a su vez, en el punto de llegada de cada nuevo proyecto. Es así como, por ejemplo, se impacta con la Bayer Ludoteca Naves durante más de tres años, entre 2005 y 2009, a partir de los hallazgos de las diversas sistematizaciones documentadas en cada lugar donde operaba el programa.

Entre los años 2009 a 2013, con el fin de identificar aprendizajes y mejorar rutinas organizacionales en la operación de un proyecto de ludotecas viajeras para llegar a la primera infancia en el marco de la atención integral, como parte de la oferta del Ministerio de Educación (MEN), y el Instituto Colombiano de Bienestar Familiar (ICBF), para 45 mil familias rurales y urbanas. Un equipo interno y externo de investigadores acompañan la sistematización desde miles de diarios de campo, innovadores aprendizajes de campo y diversas lecciones aprendidas. También se da cuenta de cómo se estaba trascendiendo de espacios fijos de ludotecas a ambientes que, desde la vida cotidiana y el entorno, se transformaban en novedosas aventuras de juego propiciadas por cerca de 2.000 profesionales cargados de pasión y entrega por su comunidad, entre nutricionistas, pedagogos y sicosociales.

La sistematización permitió documentar un libro sobre cómo se generaban vínculos afectivos sin importar si había que subirse a un caballo, burro, yegua, lancha, bus o a pie para acceder a los parques, polideportivos, escuelas rurales, canchas, casas de familia y hasta debajo de los árboles, para propiciar encuentros de aprendizaje con las familias y su primera infancia. Sistematización que corroboró que el 50 \% de las familias pasaron de o (cero) horas a un promedio de hora y media diaria de juego en familia en su casa, favoreciendo la educación inicial en el país. Impacto que trascendió a la formación de más de 800 hogares comunitarios con los que se sistematizó un modelo de atención. 
Entre 2012 y 2016, estos aprendizajes se consolidan y hacen posible que cada buena práctica documentada, al adentrarse en las teorías y disciplinas de gestión de conocimiento, permita que cada colaborador avance hacia la escritura para dejar expreso el conocimiento acumulado a partir de cada experiencia culminada. Es así como es posible aportar de manera eficiente en proyectos en el país, como Cultivarte, liderado por Banco Davivienda; diplomados sobre educación inicial, de la mano del Politécnico Grancolombiano; encuentros internacionales para transferir aprendizajes en alianza con Colsubsidio y la Gobernación de Cundinamarca; Ludociencia en poblaciones rurales, por el empeño del Grupo de Energía de Bogotá; Apúntate a Moverte, con la empresa Coca Cola y con participación en la medición de la Universidad de los Andes, para llegar a miles de escolares y docentes del país para aprender sobre hábitos saludables; Ludonutrición para hacer un seguimiento nutricional con escolares de tres ciudades, en donde su patrocinador, Fundación Femsa Mexico, le apostó a las ludotecas saludables.

Con el mismo interés por encontrar oportunidades de mejora en su quehacer, en 2016, de la mano de ICBF, OEl y el Ministerio de Vivienda, entre otros, se aportó a nuevas comunidades el conocimiento y la Metodología Naves para fortalecer la educación inicial, los entornos protectores y el tejido social. Igualmente, este conocimiento permitió convocar cerca de nueve instituciones públicas y privadas para conformar una mesa nacional que, al cabo de tres años, culminará con una propuesta de lineamientos técnicos para ludotecas en Colombia.

Cada proyecto ejecutado por la Corporación ha estado mediado por un proceso investigativo, sea con personal interno o con alianzas con la academia, como en los casos de las investigaciones de educación inicial de la Universidad San Buenaventura y la implementación de un modelo de atención para jóvenes con la Universidad Gran Colombia, y en el municipio de Icononzo, con el aporte de 


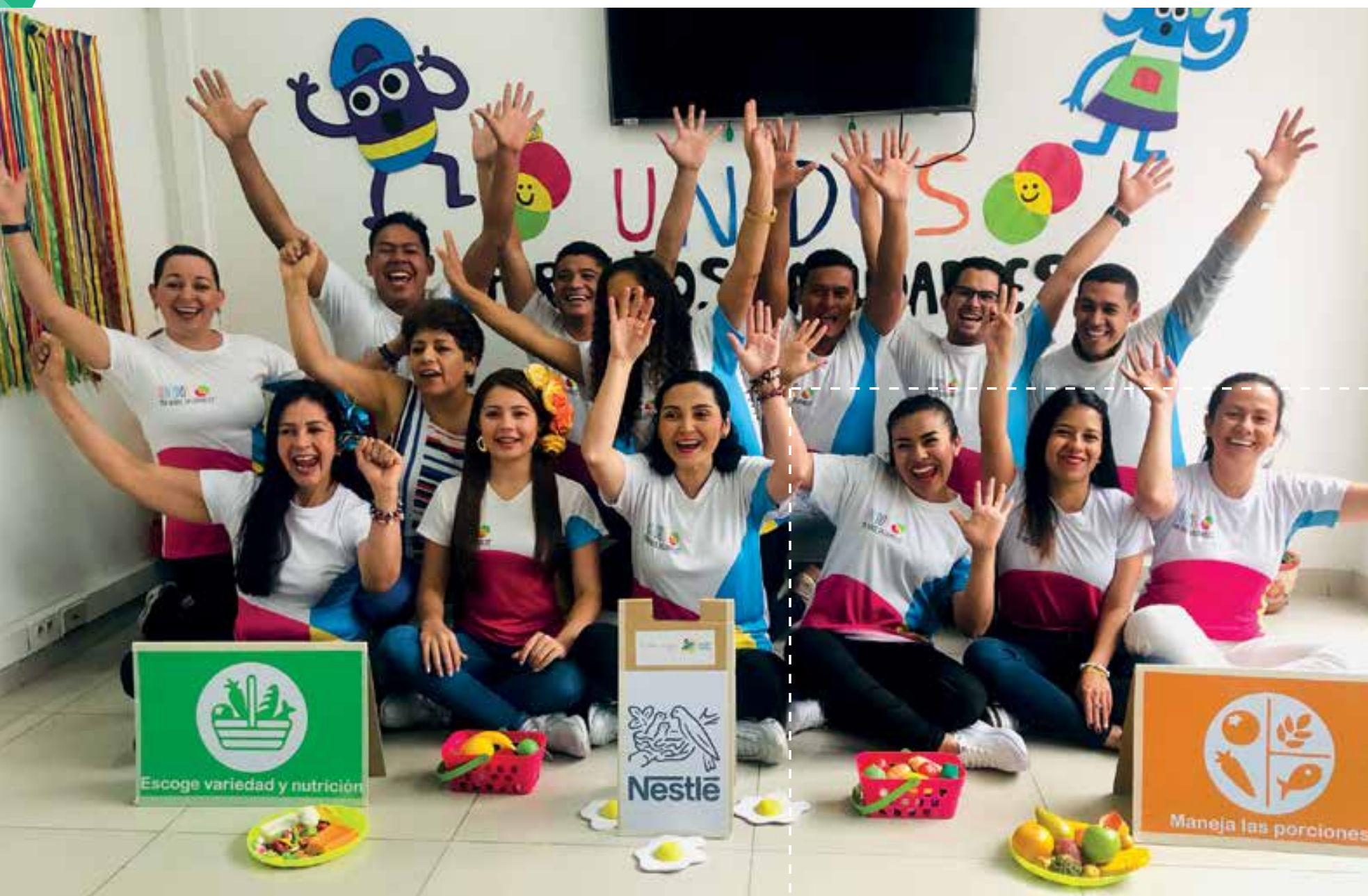

Profesionales de Nestlé Niños Saludables 
Petrobras. Muy importante también que en proyectos como Unidos por Niños Saludables, de la empresa Nestlé, operado por la Corporación desde su metodología, su impacto se haya medido por instancias académicas como la Universidad La Sabana y sus resultados nos permitan mejorar su implementación. La investigación y la gestión de conocimiento siguen siendo las mejores oportunidades que tiene la Corporación Juego y Niñez para aprender y desaprender y así con más certezas fortalecer la Metodología Naves al servicio de la asistencia técnica en modelos como este de Ludotecas Naves, que está al servicio de entes públicos y privados, en Colombia y en el mundo. Ludotecas como programas sostenibles y parte de políticas públicas de niñez y adolescencia deben ser soñadas desde la perspectiva de derechos y la investigación será la gran aliada.

\section{¿Por qué una investigación longitudinal?}

Continuando el proceso anterior, como pilar fundamental de la mejora continua de la Corporación Juego y Niñez, y como parte de su Sistema Integrado de Gestión, los órganos directivos de la Corporación Juego y Niñez comprenden la necesidad de poner nuevamente en manos de la academia especializada del país su quehacer cotidiano, y es así como en 2015 se da inicio al contrato con el Grupo de Investigación Cognición y Lenguaje en la Infancia, de la Facultad de Medicina de la Universidad Nacional de Colombia, bajo la dirección de Rita Flórez Romero, profesora e investigadora de esta universidad, con Jaime Castro Martínez, miembro del grupo de investigación, y con María Cristina Torrado y Marta Torrado, investigadoras del Observatorio de Infancia de la misma Universidad. Se inicia la primera y única investigación longitudinal sobre Ludotecas en Colombia, de manera que no solo tuviera la constatación de la eficacia de su metodología en las Ludotecas Naves, sino que hallara oportunidades de mejora continua con progresión en los proyectos en los que participa y, por ello, a partir de los resultados que estamos publicando en este texto, los años venideros serán un reto constante en su quehacer para que la calidad de la atención a los participantes

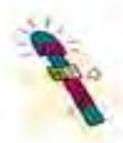


en todo el país en las Ludotecas Naves, y en cada proyecto que opera o asiste técnicamente, por parte de la Corporación Juego y Niñez, se desarrolle con los mejores estándares de calidad.

Evaluar el impacto de las ludotecas Naves en el desarrollo de competencias ciudadanas, emocionales y de creatividad le aportan al país una conversación permanente desde la reflexión, para que las acciones cotidianas que favorecen a niños y niñas en estas ludotecas de los diferentes municipios se enriquezcan y encuentren un verdadero sentido a su razón de ser. Resultados que trascenderán como aporte importante al inicio de proyectos que buscan objetivos similares para beneficiar la infancia y adolescencia en Colombia. Pesquisa que da soporte científico a un programa de educación no formal que, con estos hallazgos, se abre un camino en las políticas públicas departamentales y nacionales, y que estará a la orden de cada ambiente o territorio en donde se disponen posibilidades para que los niños y las niñas se diviertan cuando se encuentran con otros niños y niñas de su edad y su familia. Este estudio deja abierta preguntas y provocaciones para que la ciencia se formule nuevos cuestionamientos para la siguiente inspección científica sobre ludotecas y por supuesto que para La Corporación Juego y Niñez en la mejor herramienta para innovar en cada oferta de juego que propone y especialmente para que el juego en la casa, el barrio, el colegio, la biblioteca, el parque, etc, sea un asunto serio con el sentido de lo que es "Jugar para ser feliz" y no sólo para entretenerse o aprender cosas. 
